\title{
Electrospun Nanofiber Matrix with a Mucoadhesive Backing Film for Oramucosal Drug Delivery
}

\author{
Charu Tyagi, Lomas Tomar, Yahya E. Choonara, Lisa C. Du Toit, Pradeep Kumar, and Viness Pillay
}

\begin{abstract}
A rapidly disintegrating oramucosal drug delivery system was prepared with mucoadhesive polymeric backing film. Various polymers were investigated for suitability in the development of electrospun nanofibers layer and different set of polymers for backing film layer. Individual layers and the system with nanofiber spun directly on the backing film were characterized using standard techniques. Ex vivo drug permeation analysis was performed to establish the suitability of the system. Polyvinylalcohol (PVA) produced drug-loaded fibers with the most acceptable morphology and a disintegration time of $5 \mathrm{sec}$. Acceptable films casted were of PVA/hydroxypropylmethylcellulose (HPMC), with required mucoadhesive property and average disintegration time of 7-60sec. Diphenhydramine loaded delivery system exhibited 42-82\% drug permeation in study time period potentiating the feasible application of the system for oramucosal drug delivery.
\end{abstract}

Index Terms-Backing film, electrospun nanofiber matrix, mucoadhesion, oramucosa drug delivery.

\section{INTRODUCTION}

A drug delivery system intended for accelerated oramucosal drug delivery is required to be rapidly disintegrating, releasing drug almost instantaneously to the buccal mucosa for immediate absorption. This may be achieved by the use of water-soluble polymers and a large surface area exposed to the dissolution medium [1], [2]. Electrospun fibers exhibit an exceedingly large surface area to mass ratio, which not only enhances the dissolution rate, but also increases the bioavailability and total amount of drug released released and may therefore find application in rapid oramucosal drug delivery.

Due to the relatively short residence time of an oramucosally administered drug delivery system at the site of absorption, mucoadhesion is often required [3]. Mucoadhesive drug delivery systems are advantageous in that the entire system is rendered immobile, an intimate contact between the system and buccal mucosa is created and a high drug concentration at the absorption surface is achieved [3]. This results in a reduction in the required drug concentration as well as an improved bioavailability [4]. Thin, mucoadhesive films are favourable for oramucosal drug delivery due to the flexible nature and high contact surface area of such films [3]-[5].

The paper presents development of a mucoadhesive

Manuscript received July 2, 2013; revised September 1, 2013.

The authors are with University of the Witwatersrand, Faculty of Health Sciences, Department of Pharmacy and Pharmacology, 7 York Road, Parktown 2193, Johannesburg, South Africa (e-mail: charu.tyagi@wits.ac.za, yahya.choonara@wits.ac.za, lisa.dutoit@wits.ac.za, vines.pillay@wits.ac.za). polymeric backing film layer and a drug-loaded electrospun fiber layer with the aim of producing a flexible, mucoadhesive fibrous matrix system by depositing the latter directly onto the former. The film layer provided a flexible, mucoadhesive backing layer with a large exposed surface area and the drug-loaded fiber layer exhibited an exceedingly high surface area, due to the fiber dimensions, allowing for more rapid disintegration and drug release than what could be achieved from a drug-loaded film formulation. Polymers investigated for the development of the backing layer were selected based on water-solubility and film or membrane-forming propensity. The polymers investigated for the fibrous layer were selected based on water-solubility, mucoadhesiveness and electrospinnability. Diphenhydramine (DPH) was chosen as the model drug to assess the loading and release efficacy of the delivery system.

\section{MATERIALS}

Polyvinylalcohol (PVA) (87-89\% hydrolyzed, Mw 13,000-23,000g/mol), poly (ethylene oxide) (PEO), poly (acrylic acid) (PAA) (Mw 1,800g/mol) and diphenhydramine (DPH) were purchased from Sigma-Aldrich (St. Louis, Missouri, USA). Propan-2-ol, glycerol, citric acid, sodium carbonate, sodium chloride and ethanol were purchased from Rochelle Chemicals (Johannesburg, South Africa). Hydroxypropylcellulose (HPC) (Klucel Type EF and Type HF) was purchased from Hercules (Wilmington, Delaware, USA). Hydroxypropylmethylcellulose (HPMC) was purchased from Colorcon Limited (London, England).

\section{EXPERIMENTAL}

\section{A. Casting Polymeric Backing Film}

Two films were casted and investigated as backing film: PMC and the PVA/HPMC films. Polymer solutions were prepared by dissolving HPMC $(0.5-3 \% \mathrm{w} / \mathrm{v})$ or the PVA $(0.5-2.5 \% \mathrm{w} / \mathrm{v})$ and HPMC $(0-1 \% \mathrm{w} / \mathrm{v})$ in a $2: 1$ and $4: 1$ mixture of deionized water and propan-2-ol respectively. Glycerol was added to both the solutions as a plasticizer at varying concentrations of $5-70 \%$ of the polymer mass. Solutions were syringed into the respective rectangular, flat-bottomed molds and placed under an extractor at $21^{\circ} \mathrm{C}$ for 48 hours for complete solvent evaporation and film formation to occur.

\section{B. Electrospinning Nanofiber Matrix}

Solutions, employing different solvents and polymers at varying concentrations, were prepared in order to assess the electrospinning. Once appropriate solvent/polymer 
combinations were determined, diphenhydramine (DPH) was dissolved in the polymer solution. Solutions were placed in a $5 \mathrm{~mL}$ pipette, which was fitted into the adjustable supporting bracket of an electrospinning device. Electrospinning of the solutions was using a custom-built electrospinning device (RGC Engineering, Johannesburg, South Africa). Fibers were collected on aluminum foil-lined board or on prepared polymeric backing films secured to the board. Polymers investigated for electrospinning were PVA, HPC and PEO. The solution preparation and electrospinning parameters are detailed in Table I. Glycerol or glycerin was used in all electrospinning solution as the plasticizer and citric acid as a taste-masking agent in PVA solution.

\begin{tabular}{|c|c|c|c|c|}
\hline Polymer & Solvent & $\begin{array}{l}\text { Solution } \\
\text { concentrations } \\
(\% \mathrm{w} / \mathrm{v})\end{array}$ & $\begin{array}{l}\text { Tip to } \\
\text { collector } \\
\text { distance } \\
(\mathrm{cm})\end{array}$ & $\begin{array}{l}\text { Applied } \\
\text { voltage } \\
(\mathrm{kV})\end{array}$ \\
\hline PVA & W/P 2:1 & $15-30$ & $5-11$ & $15-20$ \\
\hline HPC & W/P 2:1 & 10 & $5-8$ & 20 \\
\hline PEO & Water & 10 & $7-10$ & $18-20$ \\
\hline
\end{tabular}

W/P: Deionized water/propan-2-ol

\section{Characterization}

The surface morphology of the electrospun fiber layer was analyzed by scanning electron microscopy (SEM), using a Phenom Microscope (FEI Company, Hillsboro, Oregon, USA). This was also used to confirm nanofiber formation. Rheological properties of PVA solutions employed in electrospinning were determined at $25^{\circ} \mathrm{C}$ with the use of a Haake Modular Advanced Rheometer System (ThermoFisher Scientific, Karlsruhe, Germany). The shear rate was ramped from 0 to $500 / \mathrm{s}$ and viscosity and shear force were quantified for each solution. Mucoadhesion testing was performed on backing film using a TA.XTplus Texture Analyser (Stable Micro Systems, England) fitted with a cylindrical probe. Porcine buccal mucosal tissue was attached to the probe, using rubber bands, and exposed to simulated saliva ( $\mathrm{pH}$ 6.75). The samples were attached to the stage directly below the probe. Mucoadhesion was tested by measuring the work of adhesion (WA) between the buccal mucosa and the film. The pre-test, test and post-test speeds were 2, 2 and $10 \mathrm{~mm} / \mathrm{s}$, respectively. An applied force of $50 \mathrm{~g}$, a trigger force of $5 \mathrm{~g}$ and contact time of 5 seconds were used for the test.

\section{Disintegration Time of the PVA Fiber Layer and Backing Film Layer}

The in vitro disintegration times were determined according to a modified method based on the United States Pharmacopoeia (USP) method for tablet disintegration testing using a Type PTZ 1 basket-rack assembly disintegration apparatus (Pharma Test, Hainburg, Germany). The disintegration medium was $150 \mathrm{~mL}$ simulated saliva $(\mathrm{pH}$ $6.75)$ in a glass jar placed in a water bath maintained at $37^{\circ} \mathrm{C}$. Samples were cut into sections and placed on the mesh of the basket rack assembly, with a mesh disc placed on top. The basket rack assembly was raised and lowered through a distance of $55 \mathrm{~mm}$ and at a frequency of 25 cycles per minute.

\section{E. Drug Entrapment and Ex Vivo Drug Permeation Studies}

Samples of the drug-loaded fiber layer were cut into sections, dissolved in simulated saliva ( $\mathrm{pH}$ 6.75) and the drug (DPH) content of each section was analyzed by UV spectrophotometry (Specord 40, Analytik Jena, AG, Germany), at wavelength of $254 \mathrm{~nm}$.

PVA fiber loaded with DPH, were electrospun directly onto films made up of either PVA or PVA/HPMC. The resulting fiber-on-film systems were cut into sections. Sections of freshly excised porcine mucosa tissue were mounted in Franz Type Diffusion Cells (Perme Gear, Inc., Hellertown, Pennsylvania, USA) and equilibrated for $0.5 \mathrm{~h}$ at $37^{\circ} \mathrm{C}$ by adding PBS (pH 7.4) to both the acceptor and donor compartments. After equilibration, the PBS in the donor compartment was removed and replaced with a drug-loaded sample in simulated saliva $(\mathrm{pH}$ 6.75). Samples drawn from the acceptor compartment at different time intervals were analyzed by UV spectrophotometry, and replaced by the same volume of fresh PBS.

\section{RESUlts AND DisCUSSION}

\section{A. Polymeric Backing Films}

HPMC films displayed a desirable thickness and uniformity at HPMC concentrations of $1-2 \% \mathrm{w} / \mathrm{v}$. The ideal glycerol concentration for flexible film formation was $15-30 \% \mathrm{w} / \mathrm{w}$ of the polymer mass. These films easily torned, which made removal from molds problematic. PVA/HPMC films were thin and clear or cloudy, depending on the solution constituents. PVA concentrations between 0.5 and $2 \% \mathrm{w} / \mathrm{v}$ produced films of a desirable thickness at a fill volume of $50 \mathrm{~mL}$. Above this concentration the films were too thick and fiber deposition was not uniform on these films. The optimal glycerol concentration ranged between $10-15 \% \mathrm{w} / \mathrm{w}$, below which the films were fragile and above it the films were difficult to work with. HPMC concentrations of $0-0.5 \% \mathrm{w} / \mathrm{v}$ worked well without causing breakage on removal of films from molds. The acceptable fill volume was found to be between 40 and $100 \mathrm{~mL}$. Below $40 \mathrm{~mL}$, film formation was not particularly even.

\section{B. Electrospun Nanofiber Matrix}

Electrospinning PEO solutions resulted in a thick, white layer on the collector surface. Nevertheless, fiber deposition was erratic and inconsistent having patches with great variation in thickness and even displaying clear regions. HPC electrospinning formed a fairly thick white layer on the collector, suggesting adequate fiber production. However, addition of drug hindered both fiber production and formation considerably. Therefore, no further studies were conducted on PEO and HPC for electrospinning. PVA electrospinning solutions exhibited optimum viscosity and most desirable fiber formation. The produced fibers formed a thick layer on the foil or film onto which they were electrospun, which is considered adequate for drug-loading. High plasticizer concentrations hindered the production of fibers, however lower concentrations did not interfered with 
fiber production and rather resulted in a more flexible fiber layer. Drug incorporation had little or no effect on fiber formation. Electrospun PVA fibers were therefore deemed to be satisfactory for drug delivery. The ideal PVA concentration for the electrospinning solution was determined to be $25 \% \mathrm{w} / \mathrm{v}$, the DPH concentration was $10 \% \mathrm{w} / \mathrm{v}$ and the excipients glycerol and citric acid at $5 \% \mathrm{v} / \mathrm{v}$ and $2 \% \mathrm{w} / \mathrm{v}$, respectively.

\section{Characterization}

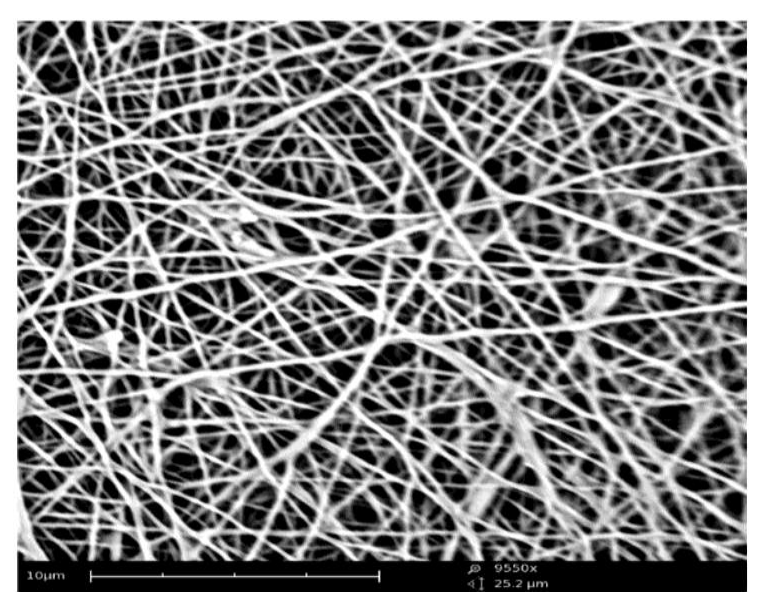

Fig. 1. Scanning electron micrograph of DPH loaded PVA fibers.

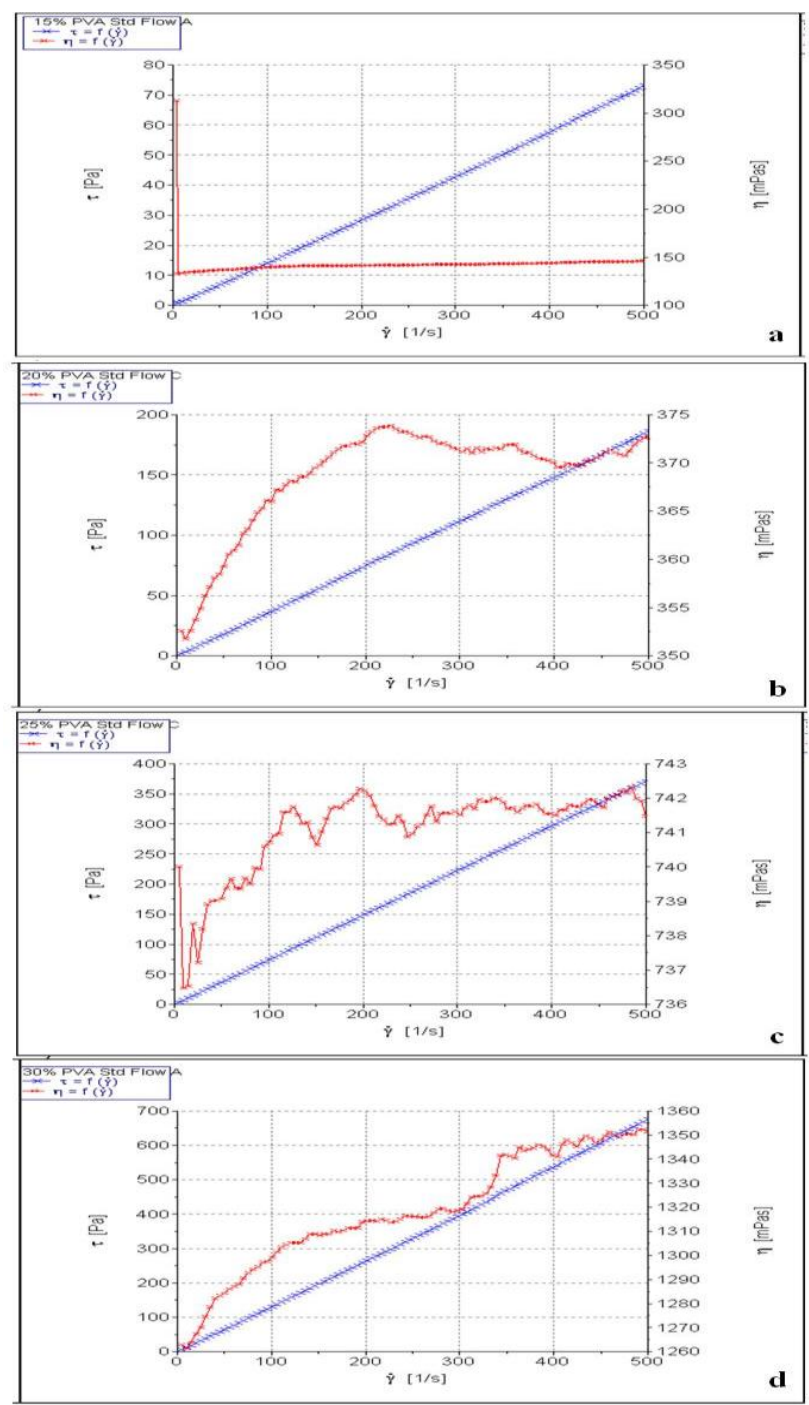

Fig. 2. Rheological profiles of (a) $15 \% \mathrm{w} / \mathrm{v}$, (b) $20 \% \mathrm{w} / \mathrm{v}$, (c) $25 \% \mathrm{w} / \mathrm{v}$ and (d) $30 \%$ w/v PVA solutions in 2:1 deionized water and propan-2-ol.
SEM analysis of PVA electrospun matrix clearly displays the presence of randomly oriented fibrous structures in the mesh (Fig. 1). Fibers formed were less than 500nm thick with somewhat uniform diameter and structure. Pores were also apparent between individual fibers.

The stress-srrain rheological parameters of a solution can have a substantial effect on the process of electrospinning as well as the quality and morphology of fibers that are formed. The degree of polymer chain entanglements, and hence the polymer concentration, has a considerable influence over the viscosity and electrospinnability of a solution. The actual conformation of individual polymer chains also has a significant influence on solution viscosity, considering that solutions containing coiled chains have a lower viscosity than those with extended chains [6]. It is therefore important to investigate the rheological properties of polymeric solutions employed in electrospinning. The rheological profiles of the $15,20,25$ and $30 \%$ w/v PVA solutions in 2:1 deionized water and propan-2-ol are depicted in Fig. 2a-d.
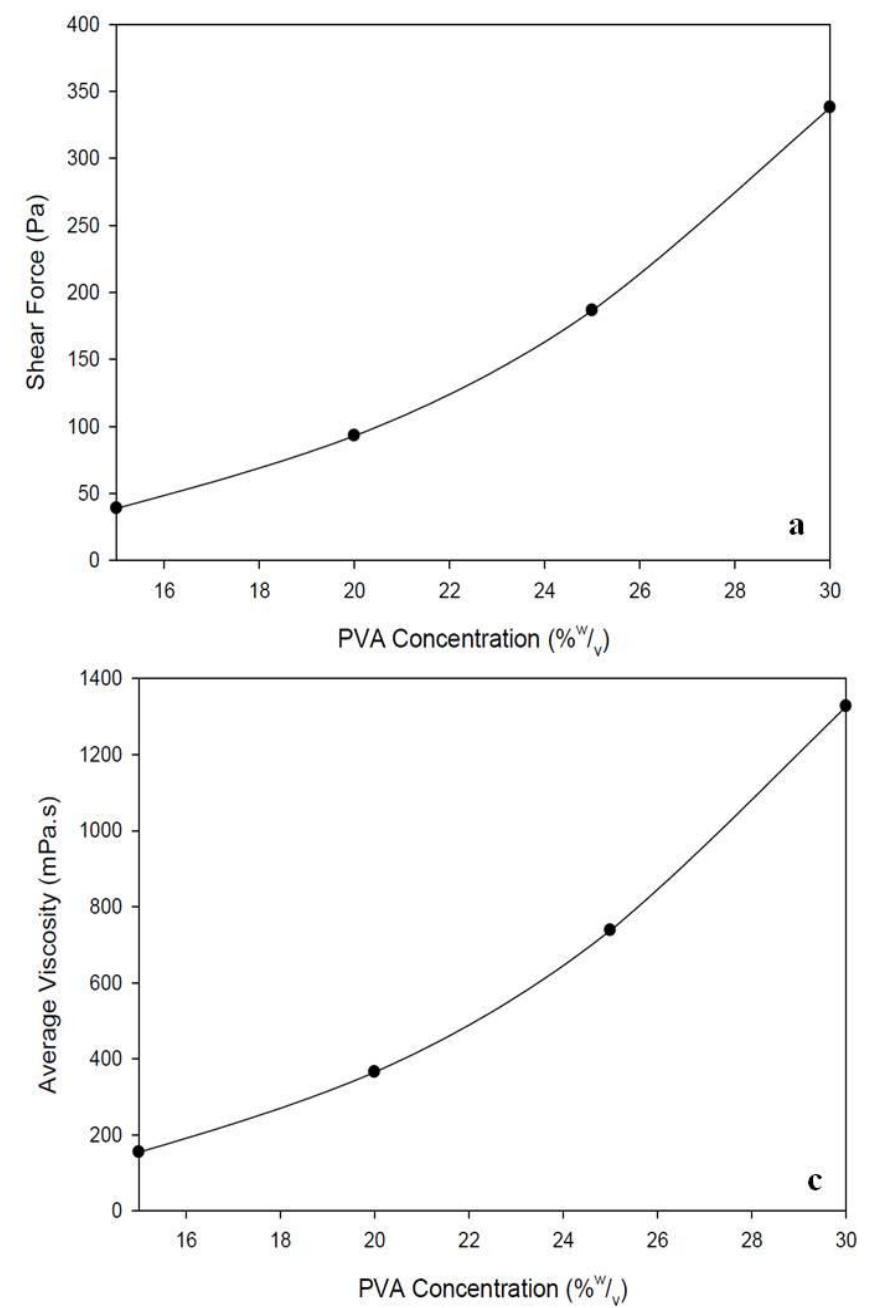

Fig. 3. Comparison for the electrospinning solutions with varying PVA concentrations (a) of average shear force; (b) of average viscosity.

The viscosity of the PVA solutions of different concentrations increased with increasing shear rate and appeared to exhibit non-Newtonian, dilatant flow properties. The average shear force $(\tau)$ and viscosity $(\eta)$, over the shear rate range, plotted against PVA concentrations is presented in Fig. 3a-b. Figures appear to display a similar shaped curve, suggesting that the increase in average viscosity with 
increasing polymer concentration is proportional to the increase in shear force. At PVA concentrations below $25 \% \mathrm{w} / \mathrm{v}$, electrospun fibers did form properly and spraying of solution droplets occurred intermittently as the viscosity was too low for constant fiber jet formation. At PVA concentrations above $25 \% \mathrm{w} / \mathrm{v}$, the solution was too viscous to pass through the capillary tip consistently. Fiber formation was satisfactory at a PVA concentration of $25 \% \mathrm{w} / \mathrm{v}$.

Mucoadhesiveness is an eminent factor to consider in oramucosal drug delivery because the retention of a drug delivery system at a specific site influences drug absorption at that site [4]. The average work of adhesion (WA) was calculated from textural profiles. It was observed that as the HPMC concentration in the formulations increased above $0.25 \%$, the average WA decreased. The process of mucoadhesion occurs largely through interpenetration and hydrogen bonding between the polymeric system and the mucosal surface. In order for interpenetration to occur between these two surfaces, the polymer chains are required to be flexible [4]. HPMC is able to form weak hydrogen bonds with mucosal surfaces and an adherent gel upon hydration, which both make it a strongly mucoadhesive polymer in theory. Notwithstanding this, it has a high glass transition temperature $\left(>200^{\circ} \mathrm{C}\right)$, which results in poor chain flexibility and therefore poor experimental mucoadhesion [7]. This elucidates the observed decrease in WA with an increase in HPMC concentration. However, formulations containing $0.5 \% \mathrm{w} / \mathrm{v}$ HPMC displayed an increase in WA with an increase in glycerol concentration. At the higher HPMC concentration, the increase in WA with increasing glycerol concentration may be attributed to the pliability of poorly-flexible HPMC chains being enhanced, hence resulting in an increased WA as the glycerol concentration was increased [8].

\section{Disintegration Time Studies}

The average disintegration time of the electrospun PVA fiber matrix was recorded as 5 seconds, which is desirable for a rapidly disintegrating drug delivery system. The average disintegration time for the backing film ranged between 7-60 seconds, depending on constituents, in the range used. To ensure high drug concentration at the absorption surface and prevent swallowing of the drug, the backing film layer is required to remain intact for a longer period of time than the fiber layer, holding fiber matrix at the absorption site.

\section{E. Drug Entrapment and Ex Vivo Drug Permeation Studies}

The average quantity of drug entrapped per $1.5 \mathrm{~cm}^{2}$ section of the electrospun fiber matrix varied according to PVA and drug concentrations in the electrospinning solutions and time of electrospinning. When these factors were increased, drug entrapment increased linearly. The average quantity of drug entrapped per section ranged between $0.3-7 \mathrm{mg}$. Drug entrapment and fiber production was observed to be greater at low humidity. Researchers have reported that as the relative humidity increases, fiber diameter decreases and bead-formation sets in because solvent evaporation is retarded at higher humidity [9]. The technique of electrospinning is therefore sensitive to environmental conditions and, hence, must be conducted in a controlled environment. Ex vivo drug permeation study determines the expediency of employing a particular DDS for buccal administration. Fig. 4 depicts the drug permeation profiles of nanofiber matrix DDS. It was observed that $42-82 \%$ of the loaded DPH dose had passed through the buccal mucosal during the testing period of $90 \mathrm{~min}$.

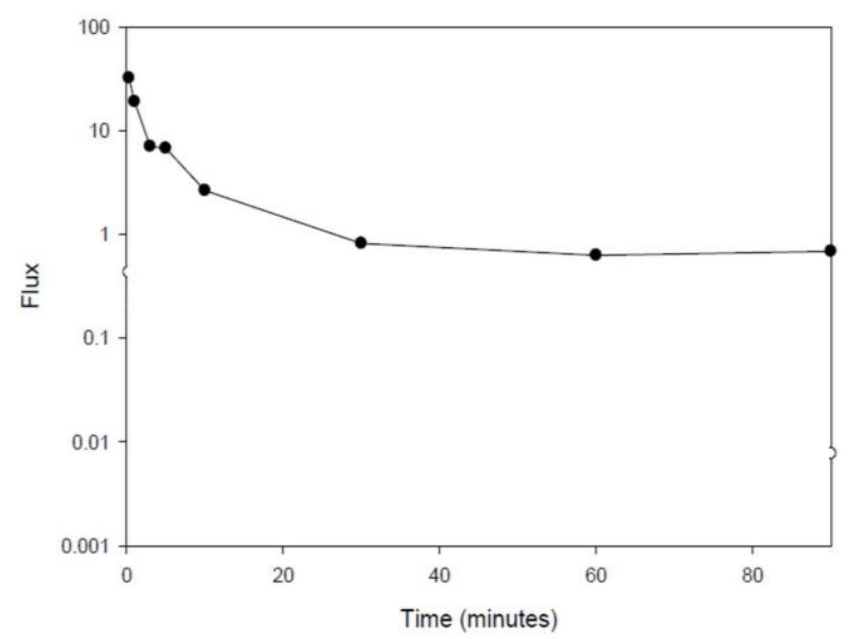

Fig. 4. Flux profiles of diphenhydramine (DPH). (in all cases $\mathrm{SDs}<0.02, \mathrm{~N}=$ $3)$.

\section{CONCLUSION}

A polymeric film layer, containing PVA, HPMC and glycerol in concentrations of $1 \% \mathrm{w} / \mathrm{v}, 0-0.5 \% \mathrm{w} / \mathrm{v}$ and $10-15 \% \mathrm{w} / \mathrm{w}$ (of total polymer mass), respectively and a fill volume of $40-100 \mathrm{~mL}$ were deemed acceptable for backing film production. Various polymers were investigated in order to develop a drug-loaded electrospun fiber layer for the system and PVA was identified as the most suitable polymer. DPH adopted as the model drug exhibited $42-82 \%$ permeation during the testing period. The ideal PVA concentration for the electrospinning solution was determined to be $25 \% \mathrm{w} / \mathrm{v}$, the drug loading concentration was $10 \% \mathrm{w} / \mathrm{v}$ and the excipients citric acid and glycerol at $2 \% \mathrm{w} / \mathrm{v}$ and $5 \% \mathrm{v} / \mathrm{v}$, respectively. This optimized electrospun nanofiber matrix system with polymer film backing proves ideal for drug delivery through oramucosa.

\section{ACKNOWLEDGMENT}

This research was funded by the National Research Foundation (NRF) of South Africa.

\section{REFERENCES}

[1] G. Verreck, I. Chun, J. Peeters, J. Rosenblatt, and M. E. Brewster, "Preparation and characterization of nanofibers containing amorphous drug dispersions generated by electrostatic spinning," Pharm Res., vol. 20, pp. 810-817, 2003.

[2] A. Dokoumetzidisa and P. Macheras, "A century of dissolution research: From noyes and whitney to the biopharmaceutics classification system," Int J. Pharm, vol. 321, pp. 1-11, 2006.

[3] G. Ponchel, "Formulation of oral mucosal drug delivery systems for the systemic delivery of bioactive materials," Adv Drug Deliv Rev., vol. 13, pp. 75-87, 1994.

[4] G. P. Andrews, T. P. Laverty, D. S. Jones, "Mucoadhesive polymeric platforms for controlled drug delivery," Eur J. Pharm Biopharm, vol. 71, pp. 505-518, 2009.

[5] L. Perioli, V. Ambrogi, F. Angelici, M. Ricci, S. Giovagnoli, M. Capuccella, and C. Rossi, "Development of mucoadhesive patches for buccal administration of ibuprofen," J. Control Release, vol. 99, pp. 73-82, 2004 
[6] S. Ramakrishna, K. Fujihara, W.-E. Teo, T.-C. Lim, and Z. Ma, "An introduction to electrospinning and nanofibers," World Scientific Publishing Co., London, 2005.

[7] E. Karavas, E. Georgarakis, and D. Bikiaris, "Application of PVP/HPMC miscible blends with enhanced mucoadhesive properties for adjusting drug release in predictable pulsatile chronotherapeutics," Eur J. Pharm Biopharm, vol. 64, pp. 115-126, 2006.

[8] J. M. Llabot, S. D. Palma, R. H. Manzo, and D. A. Allemandi, "Design of novel antifungal mucoadhesive films Part II. Formulation and in vitro biopharmaceutical evaluation," Int J. Pharm, vol. 336, pp. 263-268, 2007

[9] S. Tripatanasuwan, Z. Zhong, and D. H. Reneker, "Effect of evaporation and solidification of the charged jet in electrospinning of poly (ethylene oxide) aqueous solution," Polymer, vol. 48, pp. 5742-5746, 2007.

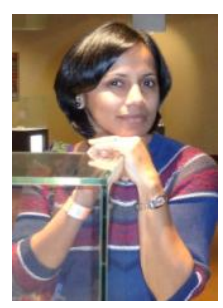

Charu Tyagi is an assisstant professor in Department of Biotechnology, V.S.P.Govt.(P.G) College, Chaudhary Charan Singh University, Ministry of Higher Education, U.P, India. She currently serves as a post-doctoral fellow in Department of Pharmacy and Pharmacology at University of the Witwatersrand, Johannesburg, South Africa. She received her Ph.D. in Biomedical Engineering from Indian Institute of Technology Delhi, New Delhi, India. She has a couple of academic and research excellence awards to her credit and is also a co-inventor of some patents. Her research interests are in the area of diagnostics and drug delivery with prominence in nano and micro-structured polymeric delivery vehicles.

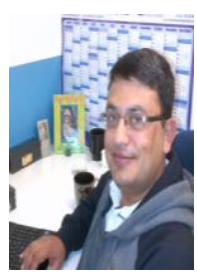

Lomas Tomar is a post-doctoral fellow in Department of Pharmacy and Pharmacology, Faculty of Health sciences at University of the Witwatersrand, Johannesburg, South Africa. He postgraduated in Biotechnology from Meerut University, Meerut, U.P, India and subsequently obtained his Ph.D. in Biomedical Engineering from Indian Institute of Technology Delhi, New Delhi, India. He earned a couple of excellence awards at scientific platform and has a few patents and international publications in peer reviewed journals. His research interests are in the area of drug delivery design, polymer synthesis, nano and microparticle preparation for smart drug deliverys in peer reviewed jour, diabetes and neurodegenerative defects.

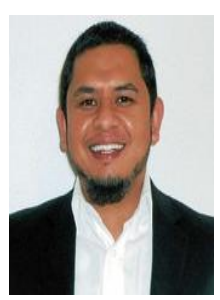

Yahya E. Choonara is an associate professor of Pharmaceutics in the Department of Pharmacy and Pharmacology at Wits University. He completed his doctorate in 'Pharmaceutics-drug delivery systems design' at the University of the Witwatersrand (Wits), Johannesburg, South Africa. He is also the research manager of the Wits Advanced Drug Delivery Platform (WADDP) funded by the Technology Innovation Agency (TIA) of South Africa.

He is a co-inventor of over 30 patents in drug delivery technology that are in various stages of prosecution in the USA, Europe, Japan and South Africa and his research has been widely published with over 85 publications in highly reputable ISI recognized scientific journals. He is a member of several prestigious academic and scientific organizations and has received number of awards in his field of research. His research interests are the fieid of drug delivery technologies, in Silico computational modeling and pharMATHeceutics.

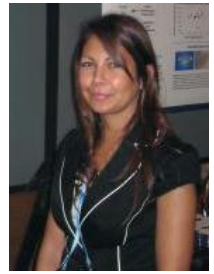

Lisa Du Toit is a lecturer of Pharmaceutical Chemistry in the Department of Pharmacy and Pharmacology, University of the Witwatersrand, Johannesburg, South Africa. She received her B.Pharm, M..Pharm from the University of the Witwatersrand, South Africa. She has been the recipient of couple of awards for the contribution in research and academic excellence. She is a co-inventor of few patents in drug delivery technology and has decent number of international publications in ISI recognized scientific journals. Her research interest focusses in the area of drug delivery design.

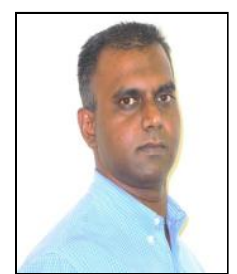

Viness Pillay is a fulbright scholar and obtained his Ph.D. from Temple University School of Pharmacy (Philadelphia, USA). He is a fellow of the African Academy of Sciences (AAS) and is a South African NRF research chair in Pharmaceutical Biomaterials and Polymer-Engineered Drug Delivery Technologies hosted by the University of the Witwatersrand. He is also a personal professor of Pharmaceutics, head of Pharmaceutics, director of the Wits Advanced Drug Delivery Platform (WADDP) and director of Pharmaceutics and Contract Research at the University of the Witwatersrand, Department of Pharmacy and Pharmacology, South Africa. He is a member of several esteemed academic associations and has to his credit hoards of prestigious scientific research awards in the field of pharmaceuticals. He has over 30 local and international patent applications currently granted or under prosecution in the USA, Europe and Japan and his research findings have been extensively published with over 130 publications in international peer-reviewed journals His research spans the areas of pharmaceutical biomaterials, nano- and micro-structured polymeric materials for controlled and smart drug delivery, innovative bioactive delivery modes and routes pertaining to various diseases and disorders like cancer, diabetes and neurodegenerative defects.

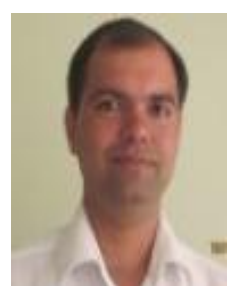

Pradeep Kumar is a lecturer of Pharmaceutics in the Department of Pharmacy and Pharmacology at University of the Witwatersrand, Johannesburg, South Africa. He received master in Pharmacy (Pharmaceutics) degree from Department of Pharmaceutical Sciences, Guru Jambheshwar University of Science and Technology, Hisar, India. $\mathrm{He}$ is a recipient of a couple of scholarships and awards and has to his credit a good number of publications in peer reviewed international journals. His research interests are in NeuroNanoPharmaceuticals, Dosage-Form-Design, biomaterials' processing, polymer synthesis, mathematical modeling for diffusional profile of solvent and drug molecules, design of experiments, SeDeM Diagrams and Isobolographic Analysis. 